\title{
Correction to: Building a Children's Health Service and System Research Strategy: development and integration in an Australian paediatric healthcare setting
}

Robyn Littlewood ${ }^{1,2}$, Oliver J. Canfell ${ }^{1,2,3^{*}}$ and Frank Tracey ${ }^{3,4}$

\author{
Correction to: BMC Health Serv Res 20, 589 (2020) \\ https://doi.org/10.1186/s12913-020-05267-6
}

Following publication of the original article [1], an error was identified in the Design section under Methods. A sentence "(3) Integration of the HSSR strategy within the health system" is placed in the wrong place. It should be placed next to "(2) Development of the CHSSR-S strategy" instead of "3. Evaluation - what are the outcomes resulting from this care?".

The updated paragraphs are given below and the changes have been highlighted in bold typeface.

Design

Development of the Children's Health Service and System Research Strategy (CHSSR-S) was informed by an inductive, bottom-up, participatory systems approach. This conceptual approach intended to place clinical, research and system leadership at the forefront of designing and implementing the CHSSR-S [16, 17]. This study was conducted across three key phases that were underpinned by our conceptual approach (see Fig. 1 for an overview of the purpose, participants and methodology of each phase):

The original article can be found online at https://doi.org/10.1186/s12913 020-05267-6.

* Correspondence: oliver.canfell@uqconnect.edu.au

'Health and Wellbeing Queensland, Queensland Government, The State of Queensland, 139 Coronation Drive, Milton, QLD 4064, Australia

${ }^{2}$ School of Human Movement and Nutrition Sciences, Faculty of Health and Behavioural Sciences, The University of Queensland, St Lucia, QLD 4067, Australia

Full list of author information is available at the end of the article
(1) Identifying the current state of HSSR within the health system

(2) Development of the CHSSR-S strategy

(3) Integration of the HSSR strategy within the health system

The overall structure and content of the CHSSR-S was aligned with three interrelated dimensions of care relevant to HSSR [10]:

1. Decision-making - what is the best care to provide?

2. Implementation - how can we best provide this care?

3. Evaluation - what are the outcomes resulting from this care?

The original article [1] has been corrected.

\section{Author details}

${ }^{1}$ Health and Wellbeing Queensland, Queensland Government, The State of Queensland, 139 Coronation Drive, Milton, QLD 4064, Australia. ${ }^{2}$ School of Human Movement and Nutrition Sciences, Faculty of Health and Behavioural Sciences, The University of Queensland, St Lucia, QLD 4067, Australia.

${ }^{3}$ Children's Health Queensland Hospital and Health Service, Department of Health, Queensland Government, The State of Queensland, South Brisbane, QLD 4101, Australia. ${ }^{4}$ Faculty of Medicine, The University of Queensland, St Lucia, QLD 4067, Australia.

Published online: 08 July 2020

\section{Reference}

1. Littlewood, et al. Building a Children's Health Service and System Research Strategy: development and integration in an Australian paediatric healthcare setting. BMC Health Serv Res. 2020;20:589. 